\title{
A Case Report of Aspergillus Endocarditis, its Complications and Associated Morbidities
}

\author{
Jian Jing Tan*, Sheldon Shao Guang Lee, Siang Chew Chai (DD and Jason Jia Hao See (iD \\ Department of Cardiology, Changi General Hospital, Singapore
}

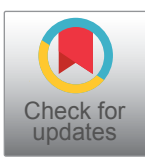

*Corresponding author: Tan Jian Jing, Department of Cardiology, Changi General Hospital, 2 Simei Street 3, 529889, Singapore

\begin{abstract}
Fungal endocarditis is a notorious disease with a high mortality rate and commonly presents with embolic phenomenon. We report a case of prosthetic valve-related Aspergillus endocarditis with aortic root pseudoaneurysm that presented with acute limb ischemia. In addition, we highlight the significant morbidity that is associated with this fungal infection. With a high clinical index of suspicion and a multidisciplinary team approach, a favourable outcome can be achieved.
\end{abstract}

\section{Case Report}

We describe a 61-year-old Chinese male who presented to a local hospital with acute right calf pain at rest. He had the cardiovascular risk factors of diabetes mellitus, hypertension, hyperlipidaemia and was status-postaortic valve replacement (Bioprosthetic Perimount Magna Ease \# 21) for severe aortic stenosis 5 months prior to presentation.

On physical examination, he was a febrile, his blood pressure was $197 / 80 \mathrm{mmHg}$, and heart rate was 50 beats per minute. He was not in respiratory distress and had an oxygen saturation of $100 \%$ on room air. His right foot appeared pale and cold, with absent pulses in the right dorsalis pedis artery and posterior popliteal artery. Sensory and motor functions were intact. There were no peripheral stigmata of endocarditis. Heart sounds were regular and no murmur was audible. Laboratory evaluation revealed unremarkable full blood count and renal panel. Urgent computed tomography (CT) angiogram of the right lower limb was performed that revealed occlusion of the popliteal artery from the level of tibial plateau to its distal portion with no flow in the anterior tibial artery (ATA), posterior tibial artery and peroneal artery (Figure 1).

Emergent surgery was performed and an embolus was found extending from the distal popliteal artery to the tibioperoneal trunk. He underwent right popliteal embolectomy, bovine patch repair, right lower limb angioplasty and anterolateral leg compartment fasciotomy. Reperfusion was successful and the embolus was sent for microbiological investigations.

On post-op day (POD) 1, our patient developed a fever and empirical intravenous Aztreonam was started. Transesophageal echocardiography(TEE) was performed that revealed an echodense mobile sessile mass $(0.8 \times 0.4 \mathrm{~cm})$ at the aortic root just distal to the origin of the right coronary artery (RCA) which was suggestive of vegetation, as well as a pseudoaneurysm in the ascending aorta (Figure 2 and Figure 3 ).

Tissue culture of the embolus revealed Aspergillus niger complex and intravenous voriconazole was started at the discretion of the Infectious Disease specialist. Ophthalmology consult was done and there was no evidence of fungal endophthalmitis. CT coronary angiography revealed 1) A pseudoaneurysm at the anterior aspect of the ascending aorta, 2) Vegetation attached to the ascending aortic wall above the origin of the RCA, 3) A tiny hypodense focus along a metallic strut of the aortic valve prosthesis, adjacent to the origin of the RCA that could represent vegetation, and 4) Possibly a subcentimetre pseudoaneurysm at the aortic valve prosthesis (Figure 4, Figure 5 and Figure 6).

The patient was transferred under the care of the

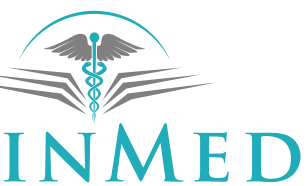

NTERNATIONAL LIBRARY
Citation: Tan JJ, Lee SSG, Chai SC, See JJH (2020) A Case Report of Aspergillus Endocarditis, its Complications and Associated Morbidities. Int J Clin Cardiol 7:170. doi.org/10.23937/2378-2951/1410170 Accepted: April 01, 2020; Published: April 03, 2020

Copyright: (C) 2020 Tan JJ, et al. This is an open-access article distributed under the terms of the Creative Commons Attribution License, which permits unrestricted use, distribution, and reproduction in any medium, provided the original author and source are credited. 


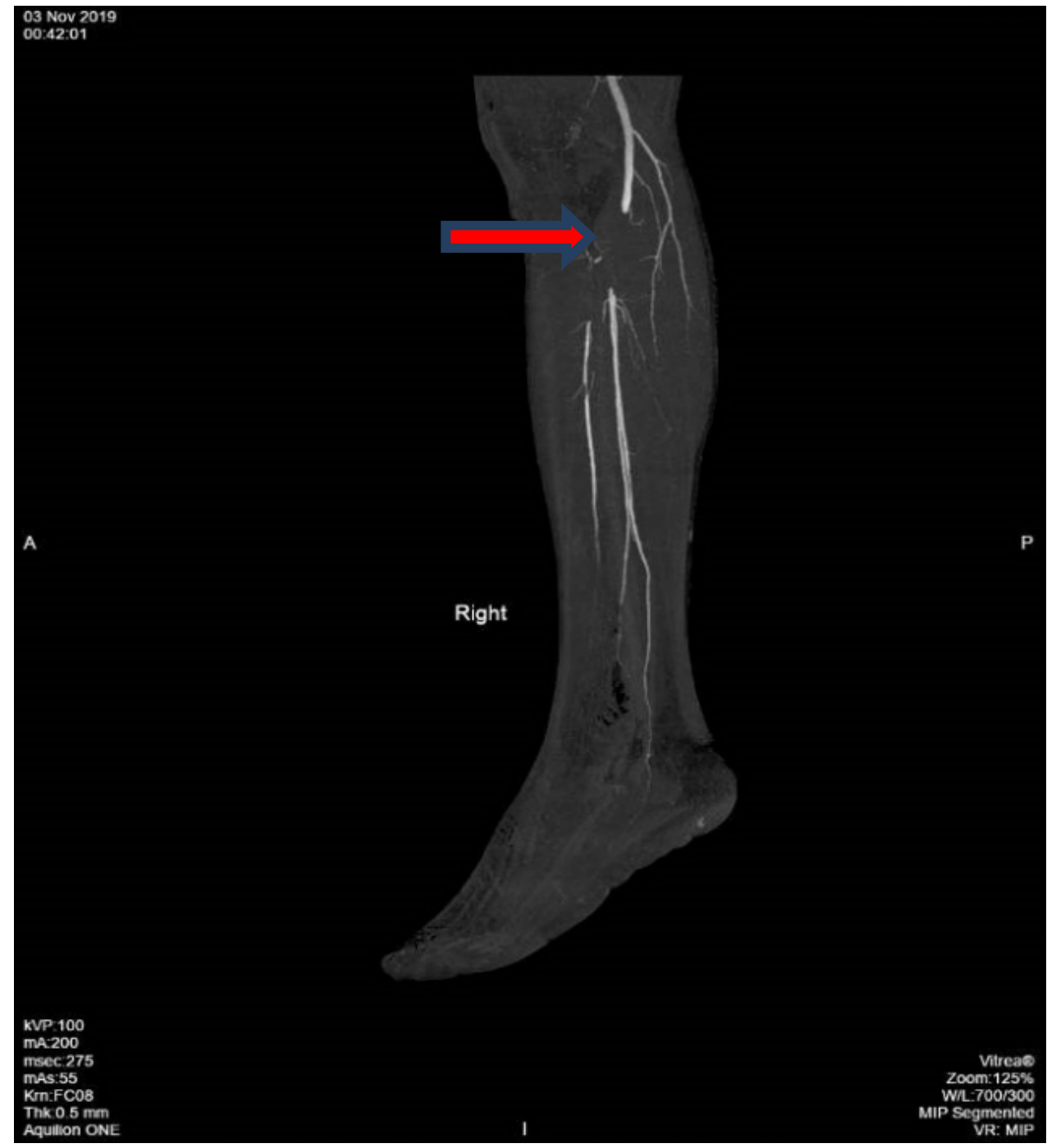

Figure 1: CT angiogram of right lower limb showing occlusion of the popliteal artery (arrow).

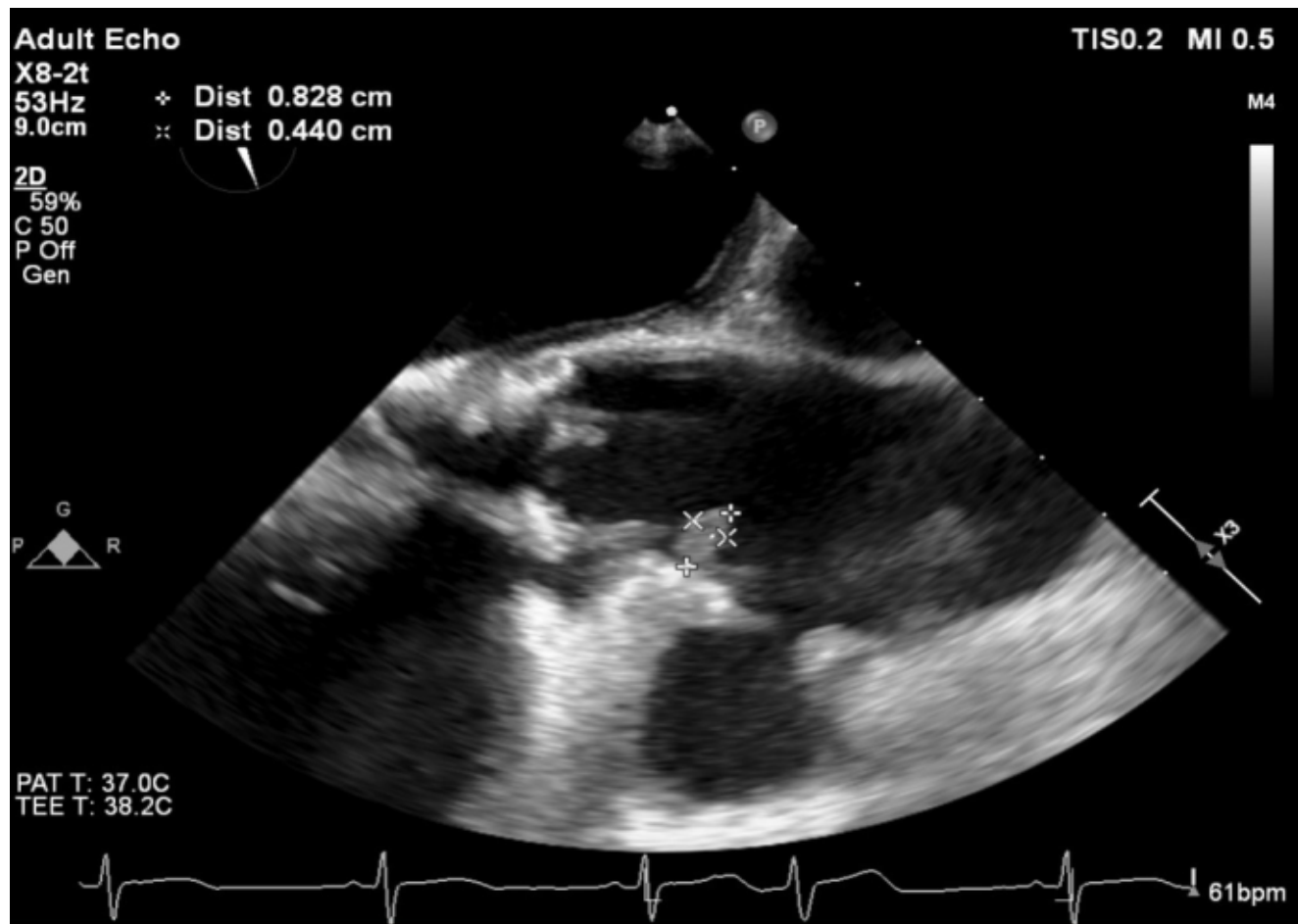

Figure 2: TEE showing an echodense mass at the aortic root just distal to the origin of the right coronary artery. 


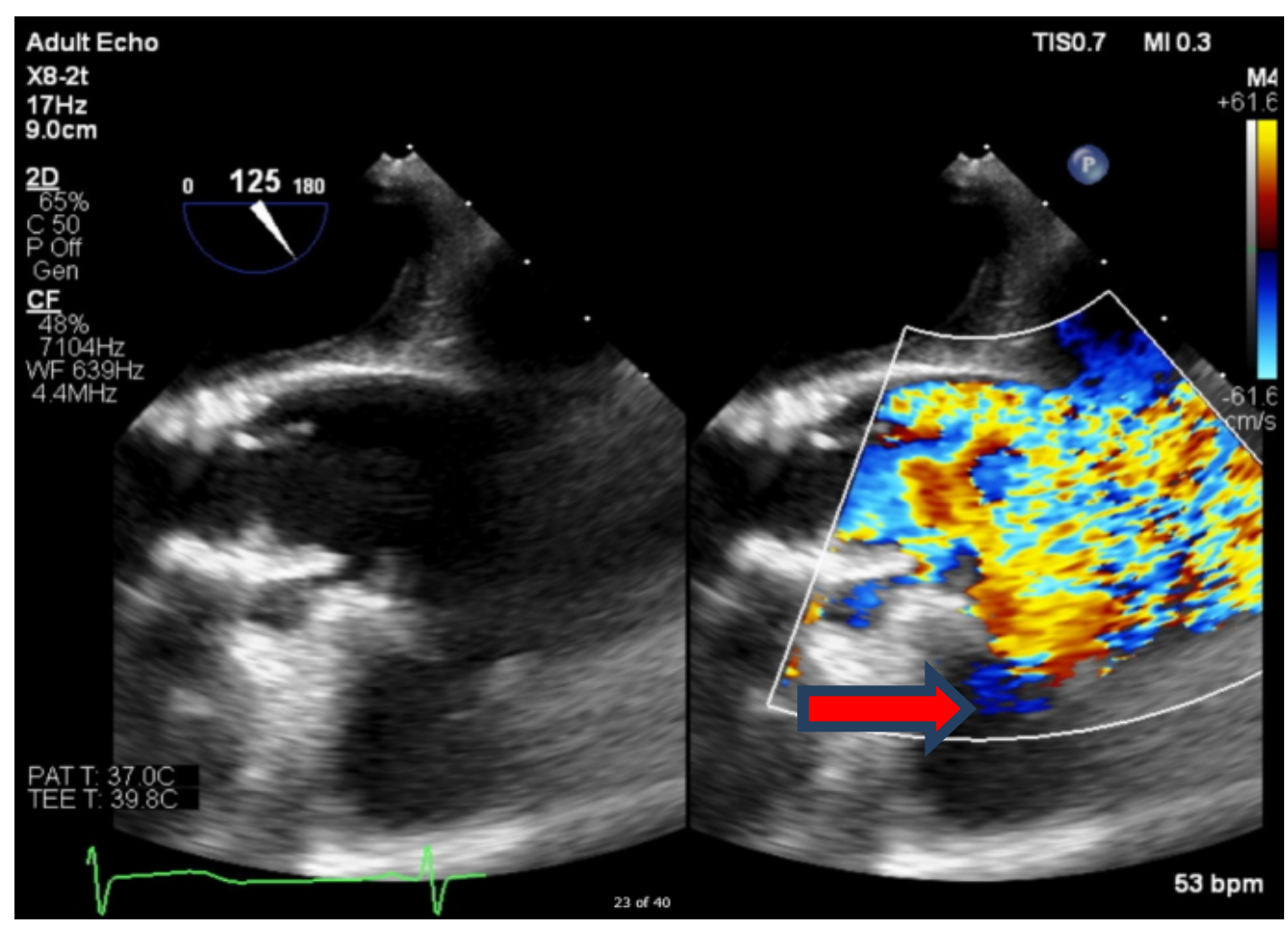

Figure 3: TEE showing a pseudoaneurysm (red arrow), distal to the mass.

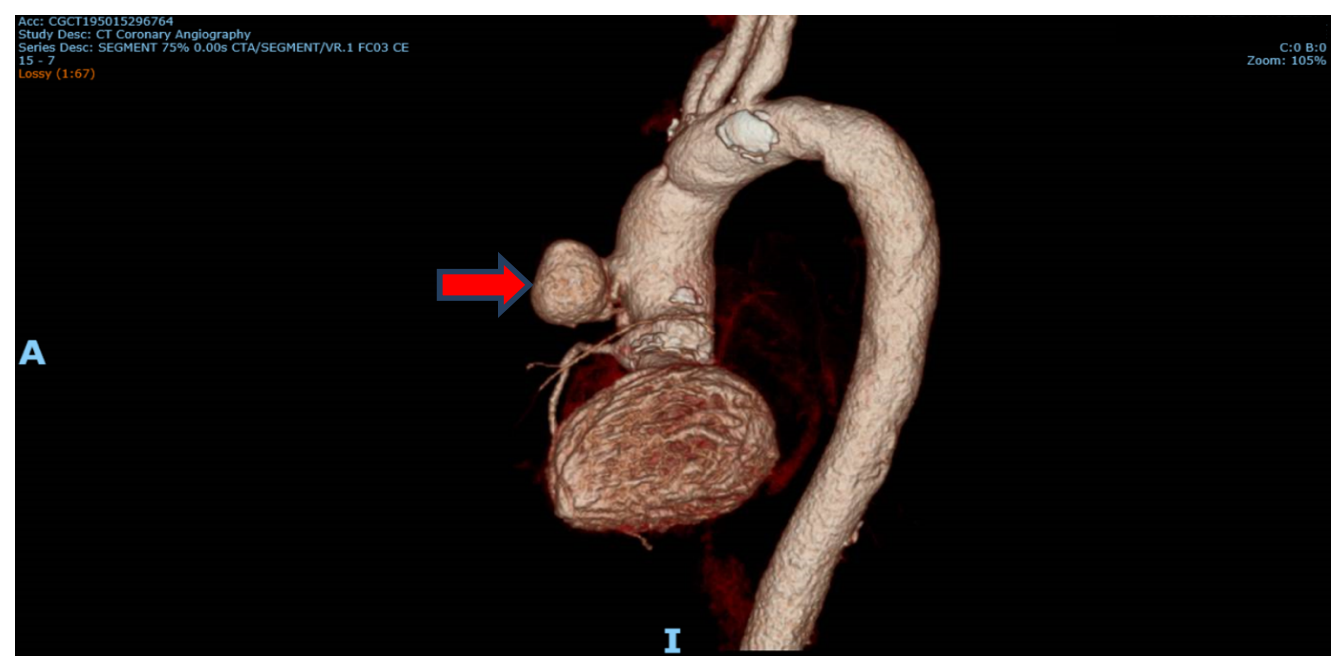

Figure 4: CT coronary angiography showing pseudoaneurysm (red arrow) anteriorly.

cardiothoracic surgery team and underwent a modified Cabrol procedure with aortic root and ascending aortic replacement (21 mm Aortic Homograft). Post-operatively, the patient developed right posterior tibial vein deep vein thrombosis (DVT) which was complicated by segmental pulmonary embolism. Subcutaneous enoxaparin was started which was subsequently switched to rivaroxaban.

About a week after his thoracic surgery, our patient started to complain of chest pain. A repeat CT thoracic aortogram showed a perigraft collection, with increased mass effect on the right pulmonary artery. There was another mediastinal collection inferiorly and an increased pericardial effusion with mass effect on the right ventricle, suspicious for cardiac tamponade. In light of these findings, he underwent emergency reopening, evacuation of clots and haemostasis. Intra-operative findings revealed large clots around the homograft and Cabrol grafts and a moderate-size pericardial effusion. Rivaroxaban was stopped and subcutaneous enoxaparin was re-instituted.

Surveillance transthoracic echocardiography (TTE) another week later showed a tiny pericardial effusion and no thrombus or vegetation was seen. Follow up CT aortogram at that juncture showed a reduction in size of the perigraft haematoma. However there were bilateral pleural effusions, with the left sided effusion being significantly larger, likely due to post-surgical inflammatory response that was managed with coop loop drainage to good effect. 


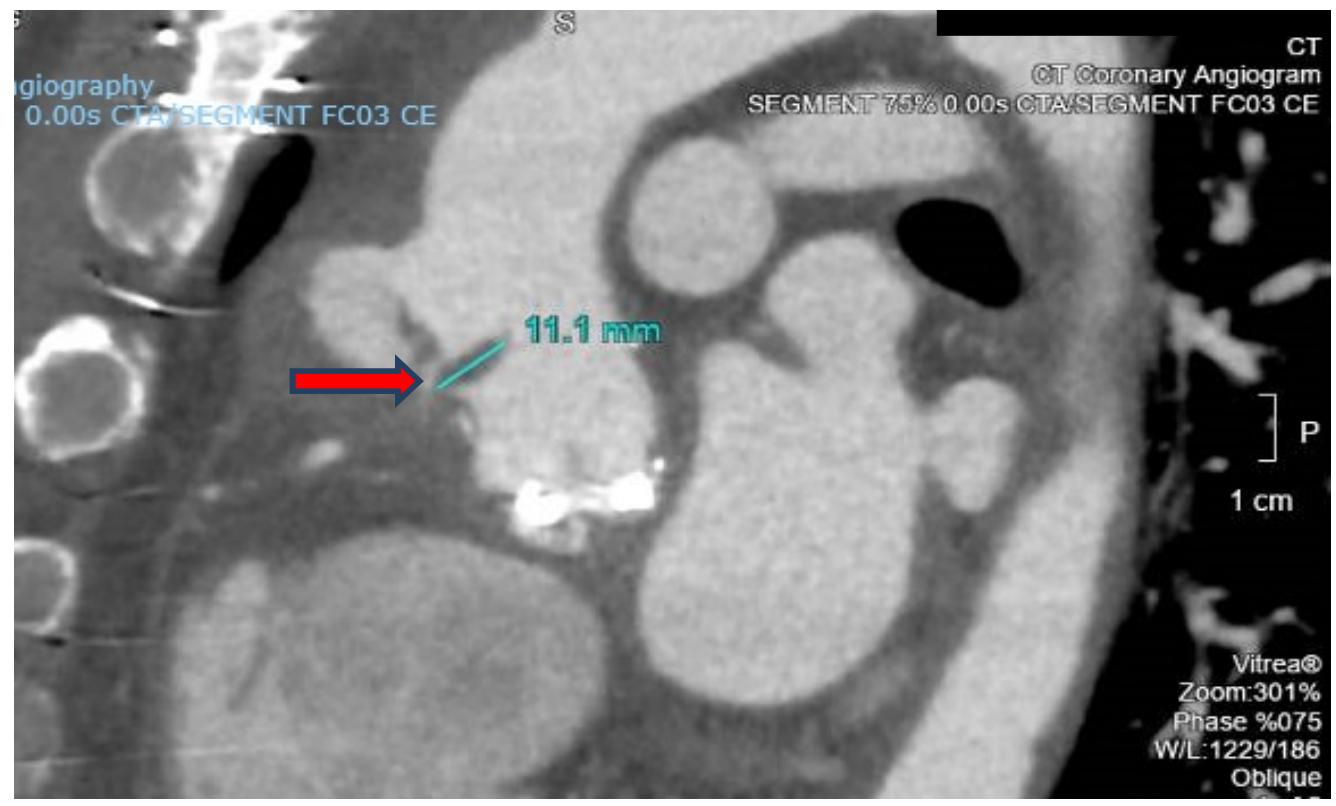

Figure 5: CT coronary angiography showing vegetation attached to the ascending aortic wall above the origin of the RCA (red arrow).

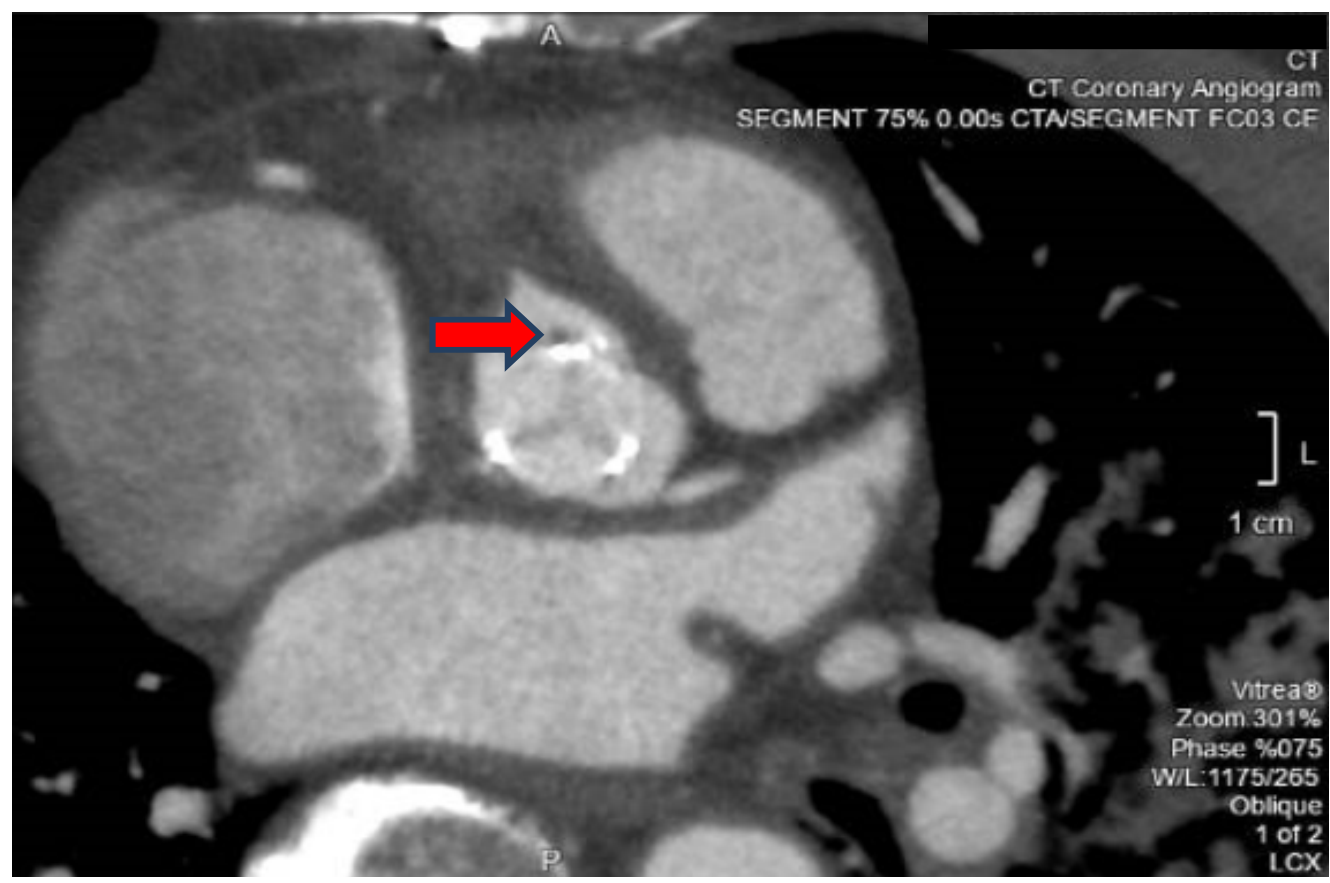

Figure 6: CT coronary angiography showing the tiny hypodense focus along the metallic prosthetic valve strut (red arrow).

He was eventually discharged nearly 2 months after his initial presentation. He remained clinically stable and a CT aortogram done one month postdischarge showed an interval decrease in the size of the perigraft haematoma with small bland residual left pleural effusion. He has remained clinically well to date during the outpatient follow up visits and will be on life-long oral voriconazole.

\section{Discussion}

Fungal endocarditis is rare and accounts for $<2 \%$ of documented cases of infective endocarditis of which only $20-25 \%$ are caused by aspergillus species [1]. As- pergillus endocarditis though uncommon has been well described in the literature as a complication of surgical valve replacement [2]. It is capable of causing sepsis, widespread embolization and multiorgan failure. Aspergillus is a ubiquitous mould found in the environment and an immunocompetent host will usually have good immunity against its spores. Before 1969, the mortality rate of aspergillosis endocarditis was near $100 \%$. In the recent years, the surgical and medical advancement in antimicrobials have significantly reduced the mortality and morbidity. Despite this, mortality rate remains at approximately $50-60 \%$ [2].

The common predisposing risk factors of an asper- 
gillus infection are an immunocompromised status, damaged lungs, prosthetic valves, rheumatic heart disease, long-term placement of a central venous catheter, total parenteral nutrition, and intravenous drug use [3]. Several reports have linked the source to be operating room air-conditioning [4].

Fungal endocarditis typically presents with fever, leukocytosis, cardiac failure or embolic complications ranging from embolic stroke, endophthalmitis to acute limb ischemia. Blood cultures are commonly negative, and diagnosis is usually made with tissue histology and culture.

The galactomannan antigen assay can be a useful adjunctive test to establish the diagnosis of invasive aspergillosis but its use has not been validated in the setting of endocarditis. It is more sensitive for detecting invasive disease due to non-fumigatus Aspergillus than A. fumigatus (49\% vs. 13\%) [5]. Pathology typically demonstrates acute angle branching and septate hyphae on Gomori methenamine silver (GMS) or periodic acid-Schiff (PAS) stains. However in our case fungal culture grew Aspergillus Niger complex and galactomannan assay was not sent.

In the choice of antimicrobials, voriconazole has been shown to be superior to amphotericin B [6-8]. This has to be coupled with timely surgical removal of the fungal source and patients will usually be placed on lifelong antifungal therapy.

In our case, the patient presented with an embolic phenomenon which is typical of fungal endocarditis. A study by Meshaal, et al. [9] found that the absence of fever, prosthetic valve endocarditis and aortic pseudoaneurysm were strong predictors of aspergillus endocarditis, clinical features that were present in our case. Mycotic aortic pseudoaneurysm is a rare complication and carries high mortality and morbidity. Its incidence is exceedingly rare, about $0.65 \%$ to $1.3 \%$ of all aortic aneurysms, with incidence of thoracic aortic aneurysms estimated to be 5.9 cases per 100,000 people/year $[10,11]$. In this case, our patient underwent immediate revascularization of the right lower limb. Upon definite diagnosis from cardiac imaging and microbiological findings, he underwent surgical intervention with the removal of the fungal mass as well as aortic root repair and prosthetic valve replacement.

Apart of concerns for survival, this case has shown significant morbidity associated with fungal endocarditis. Post aortic root repair and valve replacement, our patient suffered from impending cardiac tamponade from blood clot accumulation in the anterior mediastinum. Emergency relook sternotomy was performed for clot evacuation. Deep vein thrombosis resulted from the critical illness and prolonged bed rest has led to pulmonary embolism. Lastly patient suffered from bilateral pleural effusions from recurrent thoracic surgical manipulation, necessitating percutaneous drainage.
Lessons learnt from history and our experience have shown that a high index of suspicion must be maintained regardless of the immune status of the patient and the postoperative interval from initial valve surgery. A multi-disciplinary team approach involving the cardiologist, infectious disease physician, cardiothoracic surgeon and vascular surgeon, is vital in providing the best outcome in a patient with fungal endocarditis, and to prevent long-term sequelae.

\section{Conflict of Interest}

The authors have no conflict of interest to disclose.

\section{Source of Funding}

Not applicable.

\section{References}

1. Tattevin P, Revest M, Lefort A, Michelet C, Lortholary $O$ (2014) Fungal endocarditis: Current challenges. Int J Antimicrob Agents 44: 290-294.

2. Kalokhe AS, Rouphael N, El Chami MF, Workowski KA, Ganesh G, et al. (2010) Aspergillus endocarditis: A review of the literature. Int J Infect Dis 14: e1040-e1047.

3. Yuan SM (2016) Fungal endocarditis. Braz J Cardiovasc Surg 31: 252-255.

4. G Mehta (1990) Aspergillus endocarditis after open heart surgery: An epidemiological investigation. J Hosp Infect 15: 245-253.

5. Hachem RY, Kontoyiannis DP, Chemaly RF, Jiang Y, Reitzel $R$, et al. (2009) Utility of galactomannan enzyme immunoassay and $(1,3)$ beta-D-glucan in diagnosis of invasive fungal infections: Low sensitivity for Aspergillus fumigatus infection in hematologic malignancy patients. J Clin Microbiol 47: 129-133.

6. Herbrecht R, Denning DW, Patterson TF, Bennett JE, Greene RE, et al. (2002) Voriconazole versus amphotericin $\mathrm{B}$ for primary therapy of invasive aspergillosis. N Engl J Med 347: 408-415.

7. Thomas F Patterson, George R Thompson III, David W Denning, Jay A Fishman, Susan Hadley, et al. (2016) Practice Guidelines for the diagnosis and management of Aspergillosis: 2016 Update by the Infectious Diseases Society of America. Clin Infect Dis 63: e1-e60.

8. Gould FK, Denning DW, Elliott TS, Foweraker J, Perry JD, et al. (2012) Guidelines for the diagnosis and antibiotic treatment of endocarditis in adults: A report of the Working Party of the British Society for Antimicrobial Chemotherapy. J Antimicrob Chemother 67: 269-289.

9. Meshaal MS, Labib D, Said K, Hosny M, Hassan M, et al. (2018) Aspergillus endocarditis: Diagnostic criteria and predictors of outcome, A retrospective cohort study. PLoS One 13: e0201459.

10. Chan YC, Morales JP, Taylor PR (2005) The management of mycotic aortic aneurysms: Is there a role for endoluminal treatment? Acta Chir Belg 105: 580-587.

11. (2010) Thoracic aneurysms and aortic dissection. In: Brunicardi F, Anderson D, Dunn D, Schwartz S, Schwartz's Principles of Surgery. ( $9^{\text {th }}$ edn), McGraw-Hill Professional, New York, 668-669.
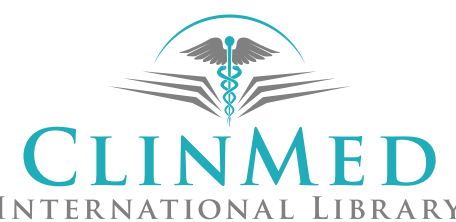